\title{
Day-ahead micro-market design for distributed energy resources
}

\author{
Pol Olivella-Rosell, Guillem Viñals-Canal \\ Andreas Sumper, Roberto Villafafila-Robles \\ Centre d'Innovació Tecnològica en Convertidors \\ Estàtics i Accionaments (CITCEA-UPC) \\ Departament d'Enginyeria Elèctrica \\ Universitat Politècnica de Catalunya, Barcelona, Spain \\ pol.olivella@citcea.upc.edu
}

\author{
Bernt A. Bremdal \\ University of Troms $\varnothing$, Narvik, Norway \\ NCE Smart Energy Markets, Halden, Norway \\ Iliana Ilieva \\ NCE Smart Energy Markets, Halden, Norway \\ Stig Ødegaard Ottesen \\ eSmart Systems, Halden, Norway
}

\begin{abstract}
This paper defines a day-ahead micro-market structure and illustrates its capability of increasing distributed energy resources' integration. This micro-market mimics in the distribution level the structure of the current European dayahead markets and their rules to introduce competition, and is based on the social welfare indicator. Micro-markets could overcome two major challenges of pool markets: they could consider the distribution network to ensure feasibility of the matched configurations and they could handle a high penetration of renewable energy without generation costs. A micro-market is controlled and supervised by the micro-market operator who executes the auction algorithm. This paper exposes a state-ofthe-art about micro-markets, proposes a structure and a set of rules, and shows micro-market's behaviour in a case study. The results show that with under-sized distribution networks the micro-market can effectively improve the social welfare with respect to other simpler approaches.
\end{abstract}

Index Terms-Distributed power generation; Energy storage; Power generation economics; power markets; micro-markets;

\section{INTRODUCTION}

Distributed generation (DG) is expected to increase in the near future [1]. Currently, small generators are aggregated for their participation in the wholesale electricity market without considering their location within the distribution network. In a high penetration of DG scenario, the power quality could decrease in terms of voltage limit violations and overloaded lines. This scenario raises two possibilities: to expand the distribution grid or to handle technical constraints with active control to manage local resources, storage systems and demand response (DR) programs. Grid expansion could be very expensive in the future due to, for example, redundant transformers [2]. Thus, it is necessary to explore control algorithms to deal with under-sized distribution grids. Moreover, electricity markets are not designed to deal with generators without generation costs [3].

Regarding the active control, different alternatives are proposed. The distribution system operator (DSO) could monitor the network variables and apply control signals to distributed energy resources (DER), such as reducing active generation or disconnecting consumption [4]. However, this alternative 978-1-4673-8463-6/16/\$31.00 (c) 2016 IEEE could compromise liberalization as the DSO criteria to take decisions is unclear.

Another option is to implement an energy management system (EMS) that coordinates DER so that the DSO needs not to worry about the grid operation. When all generators are close to each other and they can be disconnected from the main grid, it is known as micro-grid operation. EMS for micro-grids have gathered attention, under the assumption that all participants share the profits and costs of the system [5]. However, the assumption about shared profits is not realistic in systems with multiple owners spread over a distribution grid, where each participant looks for its maximum profit [6]. In those cases, solutions based on market control structures are introduced. This approach is found with different names in the literature.

Some references such as [7]-[9] use the term micro-market. The term local market is also used for the same approach in [10], [11]. However, the term local market is used for bigger systems that consider a part of the transmission system [12]-[14]. Moreover, the European Network of Transmission System Operators for Electricity (ENTSO-E) defined that in a local market area there are no transmission constraints between the market balance areas [15]. Hence, for the sake of clarity, the present work uses the term micro-market to define a market structure for distributed participants over a feeder of the distribution network.

A micro-market is an environment which allows all participants: consumers, producers and prosumers, to share their energy in a regime of competition on a distribution network level. In this marketplace generators send offers and consumers send bids, which are matched according to the clearing auction algorithm that also determines the energy prices.

The subsequent section reviews the literature about micromarket proposals. Section III exposes the structure of the day-ahead micro-market. Section IV explains the clearing algorithm implemented. Sections V and VI describe the single and multi-period problems respectively. Finally, Section VII exposes the case study analysed and Section VIII shows its results. 


\section{STATE-OF-THE-ART}

The application of electricity micro-markets at distribution level is explored by some authors. Ilic et al. presented the necessity of prosumers to trade their energy within a neighbourhood marketplace [16] and their model is based on stock exchange. Bompard et al. [17] developed a market-based control system to manage line flows considering technical limits and sending price signals to participants without a micro-market.

Regarding the participation of micro-markets in the dayahead wholesale market (DAWM), Cui et al. [18] expose the possibility that different power networks can facilitate electricity trade among neighbours participating in the DAWM so that their welfare is increased.

Another proposal for a micro-market is the one from Ampatzis et al. [19] who propose a micro-market with a trading horizon of 15 minutes and with time resolution of 5 minutes using continuous double-sided auctions. This proposal, similary to Buchmann et al. [20], includes the role of the micromarket operator (MMO). Additionally, [19] explores the cost structure of market participants.

Other authors developed new market concepts, as Nyeng et al. did in the EcoGrid project [21]. They implemented a realtime wholesale market operated by the TSO to accommodate demand response in which time resolution is 5 minutes.

Compared to the state-of-the-art, the micro-market designed in this paper makes the following contributions:

- The participation of micro-market in the day-ahead wholesale market is considered.

- Multi-period formulation considering battery state-ofcharge (SOC) maximizing total social welfare of participants is included.

- Grid constraints are managed to increase the power quality in terms of line congestions, voltage limits and grid losses.

\section{DAY-AHEAD MICRO-MARKET PROPOSAL}

The day-ahead micro-market is a market with the objective of organizing local resources using market-based rules to participate in the day-ahead wholesale market without compromising distribution networks.

The MMO is an independent entity with the aim of maximizing the profits of the community. It receives bids and offers from all participants, executes the clearing algorithm and supervises market operation similarly to the wholesale market operators.

Figure 1 compares the needed structure with and without a day-ahead micro-market. The case without a micro-market and grid constraints requires that the DSO sends signals to each agent connected to the grid to maintain grid operation feasibility. Moreover, the storage unit has to send offers and bids to the wholesale market and they might not be matched. Finally, the consumers and producers participate in the wholesale market through the retailer.

In contrast, the structure with micro-market allows participants to generate their offers and bids, and they send them to the MMO. The MMO sends feasible offers to the DAWM, and receives the prices and energy matched at the point of common coupling. The MMO uses prices to operate the storage unit and to decide set-points for participants. Figure 1 distinguishes three zones according to the SGAM methodology: Process, enterprise and market [22].

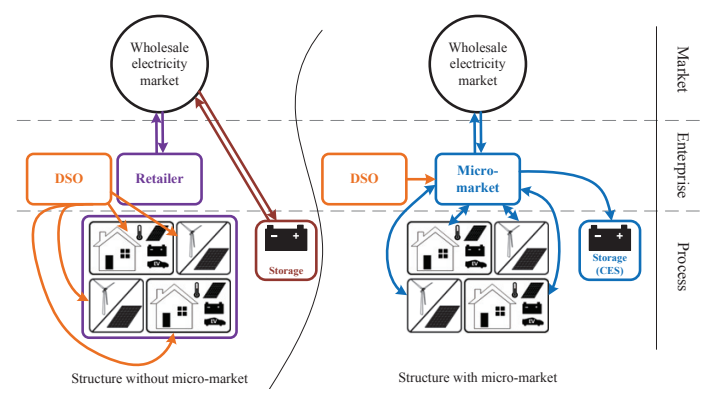

Fig. 1. Day-ahead micro-market structure proposed

The policy regulation is not considered in this work and the micro-market proposed does not consider retailers.

\section{A. Role of agents}

1) Role of $M M O$ :

- The MMO aggregates community members to take part in electricity markets. The MMO executes the clearing algorithm to determine the optimal energy to export or import depending on the DAWM price based on the micro-market participants' bids and offers.

- The MMO can use the information about the network to consider technical constraints in the micro-market clearing algorithm which is exposed in the following section.

- The MMO could offer a service of bid definition for consumers, based on their preferences, to simplify the system and this service would be optional. Furthermore, the MMO could define the generation offers based on generation forecasted for small distributed generators.

2) Role of DSO: In this proposal, the DSO has a limited responsibility; It sends the grid information to the MMO if there are changes in the network, who includes them in its clearing algorithm. Otherwise, the DSO only verifies that the technical constraints are satisfied.

3) Role of participants: Generators, consumers and prosumers have to trade the energy that they consume or produce. They have to send offers, receive auction results and to fulfil the energy settled.

4) Role of the storage: In our proposal, the storage unit is controlled by the MMO to maximize the social welfare of the micro-market community charging or discharging the battery. This assumption is based on the concept of Community Electricity Storage (CES) unit introduced by Arghandeh et al. in [23]. This paper assumes that the CES unit is owned by the community and the benefits are shared between participants. No specific remuneration is considered for the CES unit in this work. 


\section{Micro-MARKet Clearing Algorithm}

The algorithm proposed in this paper is shown in Figure 2. The algorithm can be divided in two parts, one executed before the DAWM takes place and the steps taken afterwards. Two mathematical models are proposed: The Single Period Problem (SPP) and the Multi Period Problem (MPP).

- The SPP is responsible for finding the optimal power exchanged with the main grid given a market price for each period. As the prices are not known in advance, the SPP is executed with different price scenarios to generate piece-wise offers and bids. The micro-market is considered a price-taker.

- The MPP is to be executed after the DAWM when prices are already decided. Then, the CES unit can be operated to take advantage of price differences between periods, but we may have to pay deviation costs due to difference between the power matched in the market and the power delivered eventually.

The deviation cost is assumed in this work to be $15 \%$ of the market price as an average value in the Spanish market.

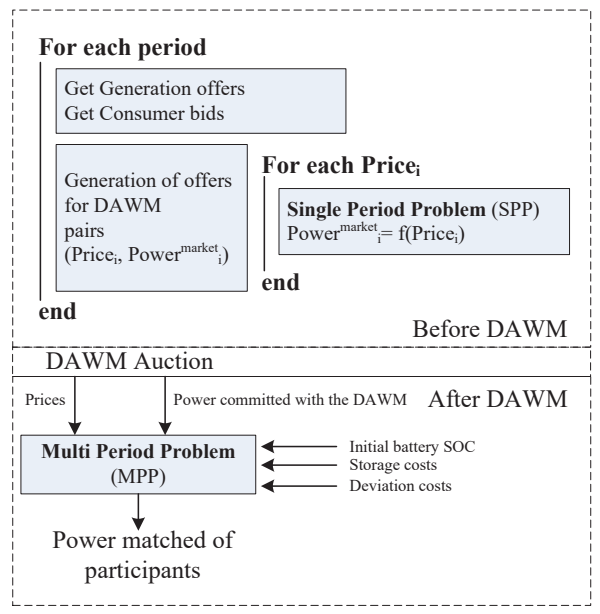

Fig. 2. Day-ahead micro-market algorithm

Once the power matched is decided, the micro-market price cannot be determined based only on matched auction curves because the following phenomena can appear:

- The energy exchanged with the main grid is paid at the DAWM price no matter the micro-market result.

- There is a price gap between the last matched offer and bid.

- There are deviation and storage costs that have to be considered.

- Nodal prices can be different if technical constrains are active in the optimal solution.

Considering the phenomena exposed previously, micromarket rules to set the price in each case have to be defined. According to the rules implemented, renewable energy generators may be promoted or consumers can pay less for their energy.

\section{Problem Formulation SPP}

In this section, parameters and variables names include superscripts while sub-indices refer to the sets over those parameters or variables are defined.

\section{A. Network}

Given a set of nodes $\mathcal{N}$ and a set of lines $\mathcal{L} \subseteq \mathcal{N} \times \mathcal{N}$ and given two indices $i, j \in \mathcal{N}$, the network impedances can be characterized with a complex matrix called admittance matrix. Let $Y_{i, j}^{m}$ and $Y_{i, j}^{\alpha}$ be the modules and angles matrices from the admittance matrix expressed in a polar form. Apparent power in lines are bounded by $S_{i, j}^{\text {maxlin }}$. Voltage modules and angles are bounded by $U^{l o}, \alpha^{l o}, U^{u p}, \alpha^{u p}$.

The variables under decision are voltages and angles at each node constrained by:

$$
U^{l o} \leq U_{i} \leq U^{u p}, \quad \alpha^{l o} \leq \alpha_{i} \leq \alpha^{u p}
$$

Active and reactive power leaving each node is presented by $P_{i}, Q_{i}$. Active and reactive power flowing from node $i \rightarrow j$ are defined as $P_{i, j}^{l i n}$ and $Q_{i, j}^{l i n}$. Those variables are constrained over the set $\mathcal{L}$ with the following equation:

$$
P_{i, j}^{l i n^{2}}+Q_{i, j}^{l i n^{2}} \leq S_{i, j}^{\operatorname{maxlin}}{ }^{2}
$$

The equations that relate voltages and angles with powers are the well-known power flow equations.

$$
\underline{\mathbf{S}}=\underline{\mathbf{U}} \cdot \underline{\mathbf{I}}^{*} \quad \underline{\mathbf{I}}=\underline{\mathbf{Y}} \cdot \underline{\mathbf{U}}
$$

\section{B. Consumers}

Consumers send bids to the MMO, those bids are step-wise cost functions of energy with the maximum price which those consumers are willing to pay.

Given a set of blocks of energy $\mathcal{B}^{c}$, a set of consumers for each node $\mathcal{D}^{c}$ and given two indices $k \in \mathcal{B}^{c}, l \in \mathcal{D}^{c}$ the offers are defined by the energy quantity, $E_{i, k, l}^{b L}$ and the price associated to that energy $P_{i, k, l}^{b L}$. Moreover, the sum of all blocks of energy is $E_{i, l}^{L}$ and the total reactive energy is $Q_{i, l}^{L}$. The power factor of the consumers is assumed constant for any matched power.

The decision variable for the consumers is the fraction of the energy of each block matched $E^{m L}$ constrained by:

$$
0 \leq E_{i, k, l}^{m L} \leq \frac{E_{i, k, l}^{b L}}{E_{i, l}^{L}}
$$

Notice that in section V-E reactive power consumed by the load will be related to $E_{i, j, k}^{m L}$.

\section{Generators}

Generators send offers to the MMO. Their offers are blocks of energy with the cost of that energy, which is equivalent to a piece-wise linear cost function. It is assumed that the MMO can send reactive power planning to the generators.

Given a set of blocks of energy $\mathcal{B}^{g}$, a set of generators for each node $\mathcal{D}^{g}$ and given two indices $m \in \mathcal{B}^{g}, n \in \mathcal{D}^{g}$ offers are defined by an energy quantity $E_{i, m, n}^{b G}$ and the cost associated $C_{i, m, n}^{b G}$ 
Reactive power is bounded by $Q_{i, n}^{\max G}$. The decision variables for generators are the power matched $E^{m G}$ and reactive power $Q^{G}$ and are constrained by:

$$
0 \leq E_{i, m, n}^{m G} \leq E_{i, m, n}^{b G}, \quad-Q_{i, n}^{\max G} \leq Q_{i, n}^{G} \leq Q_{i, n}^{\max G}
$$

\section{Common Coupling Point}

The common coupling point can act both as a consumer or generator depending on the needs of the micro-market. It is assumed that there are no bounds in power. Given a subset of grid-connected nodes $\mathcal{N}^{C C P} \subset \mathcal{N}$ and an index $o \in \mathcal{N}^{C C P}$ the decision variables of the $\mathrm{CCP}$ are $P_{o}^{C C P}, Q_{o}^{C C P}$. The price of the DAWM is defined as $C^{C C P}$. This parameter is unknown, so different scenarios are computed.

\section{E. Node Balance Equations}

The equations that relate all previous elements are known as the node balance equations. Those equations are

$$
\begin{gathered}
0=-P_{i}+\sum_{m} \sum_{n} E_{i, m, n}^{m G}-\sum_{k} \sum_{l} E_{i, k, l}^{m L} \cdot E_{i, l}^{L}+P_{i}^{C C P} \\
0=-Q_{i}+\sum_{n} Q_{i, n}^{G}-\sum_{k} \sum_{l} E_{i, k, l}^{m L} \cdot Q_{i, l}^{L}+Q_{i}^{P C C}
\end{gathered}
$$

\section{F. Objective functions}

The social welfare for a single period is defined as the sum of the generators and consumers surpluses, following [24].

$$
\begin{aligned}
& f_{o b j}^{D A W M}=-\sum_{i} \sum_{m} \sum_{n} E_{i, m, n}^{m G} \cdot C_{i, m, n}^{b G} \\
& +\sum_{i} \sum_{k} \sum_{l} E_{i, k, l}^{m L} \cdot P_{i, k, l}^{b L}-\sum_{o} P_{o}^{C C P} \cdot C^{C C P}
\end{aligned}
$$

\section{Problem Formulation MPP}

For the MPP formulation, the SPP model is defined over a new set of time periods $\mathcal{T}$. Additionally, the energy storage and the deviation cost models are included, and the objective function is modified. Consider the index $t \in \mathcal{T}$ for the following definitions.

\section{A. Energy Storage}

The energy storage unit considered in this paper is a battery. We may have several storage units but only one per node. Given a set of storage units $\mathcal{N}^{s} \subseteq \mathcal{N}$ and an index $p \in \mathcal{N}^{s}$ the battery is defined by a useful capacity considering the safe operation range of the battery $C a p_{p}^{b a t}$, an efficiency applied to the discharged energy $\eta_{p}^{s}$, reactive power capability $Q_{p}^{\max , b a t}$ and maximum active power $P_{p}^{\max , b a t}$ for both charge and discharge processes.

Additionally, storage units have an operation cost due to their loss of lifetime $C^{\text {bat }}$ which is related to the energy discharged in the objective function.

The initial state-of-charge is defined as $S_{p, 0}$. The decision variables for the energy storage units are their absorbed or generated power $P_{p, t}^{b a t A}, P_{p, t}^{b a t G}$, the state-of-charge of the battery in each period $S$ bounded by 0 and 1 .

$$
0<S_{p, t}<1
$$

The active $P^{b a t}$ and reactive power $Q^{\text {bat }}$ of the battery converter and their limits are:

$$
\begin{gathered}
-Q_{p}^{\text {max }, \text { bat }}<Q_{p, t}^{\text {bat }}<Q_{p}^{\text {max }, \text { bat }} \\
-P_{p}^{\text {max }, \text { bat }} \leq P_{p, t}^{\text {bat }} \leq P_{p}^{\text {max }, \text { bat }}
\end{gathered}
$$

The equations that define the battery behaviour are the SOC relation:

$$
S_{p, t}=S_{p, t-1}+\frac{1}{C a p_{p}^{b a t}} \cdot\left(P_{p, t}^{b a t A}-\frac{P_{p, t}^{b a t G}}{\eta_{p}^{s}}\right)
$$

\section{B. Market Contract}

After we have a matched power $P_{o, t}^{m a t}$ to exchange with the main grid from the DAWM, we can choose not to deliver or consume that power at the expense of paying a penalization $\operatorname{cost} C_{t}^{\text {dev }}$ depending on the market price.

The absolute value of the deviation $D v^{a b s}$ is defined with the equations:

$$
\begin{aligned}
& D v_{o, t}^{a b s} \geq P_{o, t}^{C C P}-P_{o, t}^{\text {mat }} \\
& D v_{o, t}^{a b s} \geq P_{o, t}^{\text {mat }}-P_{o, t}^{C C P}
\end{aligned}
$$

$D v^{a b s}$ and $C^{d e v}$ will be included in the objective function to compute the deviation costs.

\section{Objective Function}

When the energy storage is considered, we want to maximize the sum of social welfare over all periods, even if some participants may be disadvantaged in certain periods. The objective function also includes the deviation costs and the operation and maintenance cost of the battery.

$$
\begin{gathered}
f_{o b j}^{C E S}=-\sum_{t} \sum_{i} \sum_{m} \sum_{n} E_{i, m, n, t}^{m G} \cdot C_{i, m, n, t}^{b G} \\
+\sum_{t} \sum_{i} \sum_{k} \sum_{l} E_{i, k, l, t}^{m L} \cdot P_{i, k, l, t}^{b L} \\
\quad-\sum_{t} \sum_{o} P_{o, t}^{C C P} \cdot C_{t}^{C C P} \\
-\sum_{p} \sum_{t} P_{p, t}^{b a t G} \cdot C_{p}^{b a t}-\sum_{t} \sum_{o} D v_{o, t}^{a b s} \cdot C_{t}^{\text {dev }}
\end{gathered}
$$

Notice that, $E^{m G}$ and $E^{m L}$ are variables because the usage of the CES unit may alter the power matched of the micromarket's participants.

\section{CASE STUDY}

In this section, the case study is presented. It includes photovoltaic (PV) producers, prosumers with rooftop PV panels and consumers without generation connected to a meshed distribution network. The demand is assumed elastic because consumers may have demand-response capability or remotely controllable electric vehicles.For the MPP, five periods of one 
hour are studied to illustrate the micro-market behaviour.

The micro-market results are compared to the case without a micro-market in order to assess its benefits. When there is not a micro-market, power matched in the DAWM might violate some grid constraints. In this work it is assumed that in those cases the DSO monitors the grid. When the DSO detects violations, applies power curtailments sending an active power reduction signal to all generators until the violation is corrected. This signal is a relative reduction of power and it is the same for all generators.

Figure 3 shows the energy offered by all PV generators, the maximum energy demanded by micro-market's participants, and the DAWM price. During the initial periods the micromarket has energy surplus and during the last hours energy deficit. Moreover, the grid price is cheaper during initial periods than final periods.

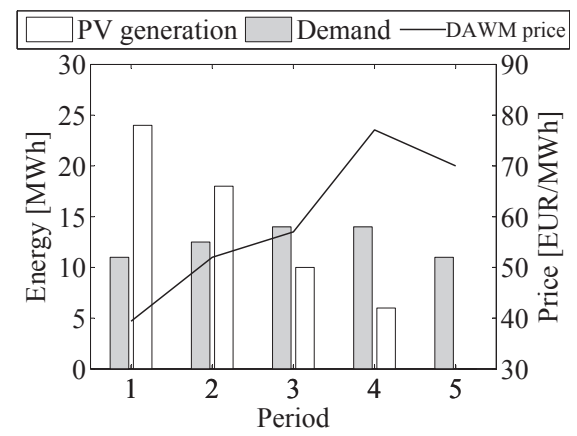

Fig. 3. Case study data

\section{A. Network model}

In order to show the DAMM operation, the case study analysed is a 4 node distribution network with a high penetration of renewable generation shown in Figure 4. The CES unit is connected to node 4 with a charging/discharging capacity of $1 \mathrm{MW}$ and \pm 1 MVar, $3 \mathrm{MWh}$ of useful energy and $85 \%$ of full cycle efficiency.

As it has been mentioned earlier, the grid is only under-sized for peak generation power, not for the consumption; the line between bus 1 and 2 has 12 MVA capacity which constraints the power exchange with the main grid, the other lines cannot export the full power of renewable generation of G3 and G4 which is $12 \mathrm{MW}$ peak each one.

\section{B. Simulation cases}

1) Without micro-market: DER units are aggregated for the participation in the DAWM without considering the grid. During the operation, corrections needed to avoid grid violations are determined by the DSO's distribution management system.

2) With micro-market: DER units are aggregated for the participation in the DAWM considering the grid.

3) With micro-market and CES unit: This case shows the effect of the battery unit on the participants' social welfare.

\section{RESULTS}

In this section, results are presented for the three cases simulated. Table II shows a Social Welfare Comparison. During the first periods considered there is a great amount of renewable generation and, as the grid is under-sized, the renewable power cannot be exported to the main grid without overcharging lines 1-2, 4-2 and 3-2. Without a micro-market, the DSO is forced to reduce power of all generators as it is shown in Table I. If the reduction of generation makes it not possible to satisfy all consumption, the main grid acts as slack bus. This is far less efficient than considering the network during the clearing algorithm execution. In the last periods, when there is little renewable generation, the micro-market benefits without a battery are not significant.

TABLE I

REDUCTION SIGNAL FOR CASE 1

\begin{tabular}{llllll}
\hline Period & 1 & 2 & 3 & 4 & 5 \\
Reduction signal & 0.6 & 0.7 & 1 & 1 & 1 \\
\hline
\end{tabular}

TABLE II

SOCIAL WELFARE OF EACH PERIOD AND SIMULATED CASES

\begin{tabular}{crrr}
\hline Period & \multicolumn{3}{c}{ Simulation case } \\
& \multicolumn{1}{c}{$\mathbf{1}$} & \multicolumn{1}{c}{$\mathbf{2}$} & \multicolumn{1}{c}{$\mathbf{3}$} \\
\hline $\mathbf{1}$ & $1,446.1$ & $1,576.7$ & $1,599.2$ \\
$\mathbf{2}$ & $1,713.9$ & $1,848.3$ & $1,881.5$ \\
$\mathbf{3}$ & $1,813.2$ & $1,815.1$ & $1,817.3$ \\
$\mathbf{4}$ & 1,591 & $1,593.2$ & $1,661.1$ \\
$\mathbf{5}$ & 842.3 & 843.6 & 861.8 \\
\hline total & $7,406.5$ & $7,676.9$ & $7,820.9$ \\
$\Delta(\%)$ & 0 & 3.651 & 5.595 \\
\hline
\end{tabular}

When a CES is considered, the overall SW is increased. In this simulation it is forced that the battery ends with the initial SOC to avoid free energy injections. The battery is capable of increasing the social welfare in all periods, and not only in those periods where it acts as generator. In order to understand the battery participation, the period 2 is studied in more detail for the case with micro-market and CES and shown in Figure 4.

As the Figure 4 shows, during period 2 the CES unit stores energy and produces reactive power locally to increase the active power transmission capacity. Furthermore, lines between nodes 2-3 and 2-4 are near to their limit and for this reason, G3 and G4 are reduced from their maximum power.

Figure 5 shows the auction curves for the other periods. During periods 1 and 2 not all renewable generation can be matched and the battery stores energy. In contrast, the battery does not act during period 3 because the DAWM price is lower than periods 4 and 5 . During these periods the battery delivers the energy stored to reduce the power consumed from the main grid.

\section{COnClusions}

In this paper a micro-market is introduced to manage DER. With the increase of renewable distributed generation the current network can be under-sized. If the network is not considered for the participation of DER to the DAWM, power 


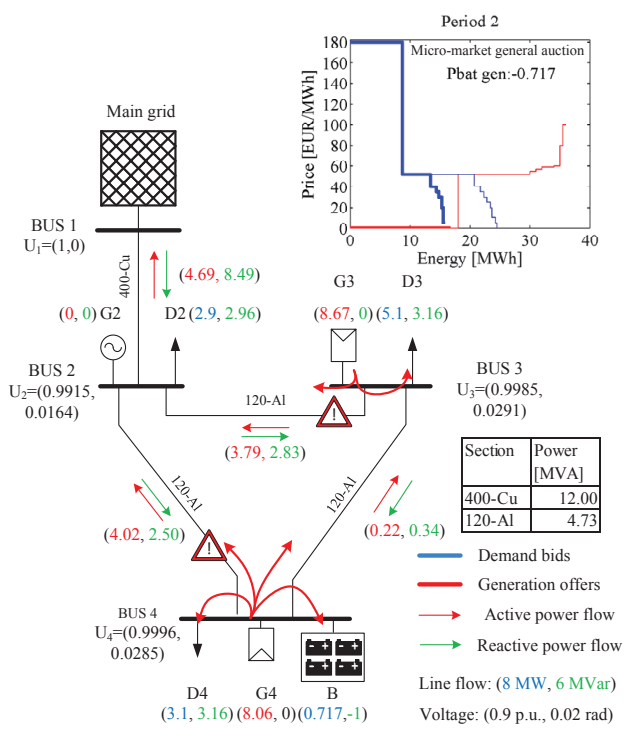

Fig. 4. Period 2 results for case 3
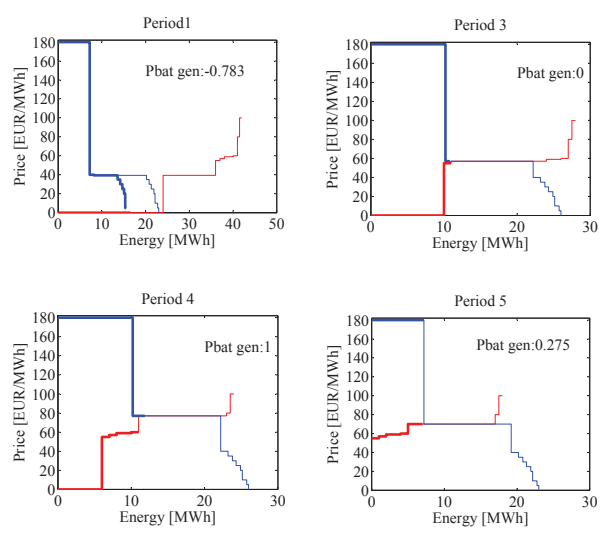

Fig. 5. Auctions for case 3

quality can be compromised and eventually power can be curtailed in a non-optimal way. The micro-market structure presented ensures competitiveness among agents considering social welfare in its clearing algorithm. The micro-market proposed can help both ensure the network constraints and increase the social welfare, especially when renewable energy is high. Moreover, a CES unit can be added to improve the performance of the micro-market, and it can effectively increase the social welfare.

\section{Acknowledgements}

This work was supported by the KIC Innoenergy $\mathrm{PhD}$ School and by the European Union's Horizon 2020 EMPOWER project under grant agreement No 646476.

\section{REFERENCES}

[1] International Energy Agency (IEA). Medium-Term Renewable Energy Market Report 2015. Market Analysis and Forecasts to 2020. Technical report, 2015.

[2] Rahmatallah Poudineh and Tooraj Jamasb. Distributed generation, storage, demand response and energy efficiency as alternatives to grid capacity enhancement. Energy Policy, 67:222-231, 2014.
[3] M Ahlstrom, E Ela, J Riesz, J O’Sullivan, B F Hobbs, M O’Malley, M Milligan, P Sotkiewicz, and J Caldwell. The Evolution of the Market: Designing a Market for High Levels of Variable Generation, 2015.

[4] Andre Madureira, Clara Gouveia, Carlos Moreira, Luis Seca, and Joao Pecas Lopes. Coordinated management of distributed energy resources in electrical distribution systems. In 2013 IEEE PES Conference on Innovative Smart Grid Technologies (ISGT Latin America), pages 18. IEEE, apr 2013.

[5] S Parhizi, H Lotfi, A Khodaei, and S Bahramirad. State of the Art in Research on Microgrids: A Review. Access, IEEE, 3:890-925, 2015.

[6] Christine Schwaegerl and Liang Tao. The Microgrids Concept. In Microgrids, pages 1-24. John Wiley and Sons Ltd, 2013.

[7] Isaac Faber, William Lane, Wayne Pak, Mary Prakel, Cheyne Rocha, and John V Farr. Micro-energy markets: The role of a consumer preference pricing strategy on microgrid energy investment. Energy, 74:567-575, 2014.

[8] Alexandru Kriukov. Applying a Micro-Market Inside an Electric Vehicles Parking Facility. In Power Engineering Conference (UPEC), 2014 49th International Universities, 2014.

[9] Cadets William Lane, Wayne Pak, Mary Prakel, Cheyne Rocha, M A J Isaac Faber, and John V Farr. Costing Consumer Preferences for a Micro Energy Market. Technical Report July, Center for nation reconstruction and capacity development, 2013.

[10] D Menniti, A Pinnarelli, N Sorrentino, A Burgio, and G Belli. Management of storage systems in local electricity market to avoid renewable power curtailment in distribution network. In Power Engineering Conference (AUPEC), 2014 Australasian Universities, pages 1-5, 2014.

[11] C Rosen and R Madlener. An auction mechanism for local energy markets: Results from theory and simulation. In Complexity in Engineering (COMPENG), 2012, pages 1-4, 2012.

[12] Nan-Peng Yu, Chen-Ching Liu, and J Price. Evaluation of Market Rules Using a Multi-Agent System Method. Power Systems, IEEE Transactions on, 25(1):470-479, 2010.

[13] W Kamrat. Modeling the structure of local energy markets. Computer Applications in Power, IEEE, 14(2):30-35, 2001.

[14] Yunpeng Wang, W Saad, Zhu Han, H V Poor, and T Basar. A GameTheoretic Approach to Energy Trading in the Smart Grid. Smart Grid, IEEE Transactions on, 5(3):1439-1450, 2014.

[15] ENTSO-E. The harmonised electricity market role model, 2011.

[16] D Ilic, P G Da Silva, S Karnouskos, and M Griesemer. An energy market for trading electricity in smart grid neighbourhoods. In Digital Ecosystems Technologies (DEST), 2012 6th IEEE International Conference on, pages 1-6, 2012.

[17] Ettore F. Bompard and Bei Han. Market-based control in emerging distribution system operation. IEEE Transactions on Power Delivery, 28(4):2373-2382, 2013.

[18] Tiansong Cui, Yanzhi Wang, Shahin Nazarian, and Massoud Pedram. An electricity trade model for multiple power distribution networks in smart energy systems. In PES General Meeting-Conference \& Exposition, 2014 IEEE, pages 1-5. IEEE, 2014.

[19] Michail Ampatzis, Phuong H Nguyen, and Wil Kling. Local electricity market design for the coordination of distributed energy resources at district level. In Innovative Smart Grid Technologies Conference Europe (ISGT-Europe), 2014 IEEE PES, pages 1-6. IEEE, 2014.

[20] Erik Buchmann, Stephan Kessler, Patrick Jochem, and Klemens Bohm. The costs of privacy in local energy markets. In Proceedings - 2013 IEEE International Conference on Business Informatics, IEEE CBI 2013, pages 198-207, 2013.

[21] Preben Nyeng, Koen Kok, Salvador Pineda, Ove Grande, Jonathan Sprooten, Bob Hebb, and Frans Nieuwenhout. Enabling demand response by extending the European electricity markets with a real-time market. In 2013 4th IEEE/PES Innovative Smart Grid Technologies Europe, ISGT Europe 2013, pages 1-5, 2013.

[22] Smart Grid Coordination CEN-CENELEC-ETSI. Smart Grid Reference Architecture. Technical report, 2012.

[23] Reza Arghandeh, Jeremy Woyak, Ahmet Onen, Jaesung Jung, and Robert P Broadwater. Economic Optimal Operation of Community Energy Storage Systems in Competitive Energy Markets. Applied Energy, 135:1-17, 2014.

[24] Jiang Wu, Xiaohong Guan, Feng Gao, and Guoji Sun. Social welfare maximization auction for electricity markets with elastic demand. Intelligent Control and Automation, 2008. WCICA 2008. 7th World Congress on, pages 7157-7162, 2008. 